\title{
AN EXTREMAL PROBLEM FOR FUNCTIONS WITH POSITIVE REAL PART
}

\author{
R. S. GLPTA
}

\begin{abstract}
Let $\mathscr{P}$ be the class of functions $P(z)$, normalized so that $P(0)=1$ which are regular in $|z|<1$ and have positive real part there. We obtain the minimum (maximum) real part of $e^{i \alpha}\left(z P^{\prime} \mid P\right)$ for fixed $\alpha$ and $|z|, \alpha \in[0,2 \pi],|z|<1$ and $P$ running over the class $\mathscr{P}$.
\end{abstract}

1. Introduction. Let $\mathscr{P}$ denote the class of functions $P(z)$, normalized so that $P(0)=1$ which are regular in $E=\{z:|z|<1\}$ and have positive real part there. The class $\mathscr{P}$ occupies an extremely important place in the theory of functions and as such it has been studied by several authors ([1], [2], [3], [4], [5]).

In the present paper we propose to obtain the minimum (maximum) of the real part of $e^{i \alpha}\left(z P^{\prime} \mid P\right)$ for fixed $\alpha$ and $|z|, \alpha \in[0,2 \pi], z \in E$ and $P$ running over the class $\mathscr{P}$. The corresponding results for $\alpha=0$ are well known and are frequently applied in extremal problems connected with univalent and multivalent functions. Our results determine, in particular, the region of variability of $z P^{\prime} \mid P, P \in \mathscr{P}$. We make use of a theorem of Zmorovič [6] and combine it with Sakaguchi's variational formula for (5]. Our technique is novel and capable of solving a variety of extremal problems. Some of these are presented elsewhere.

2. Preliminary. Throughout this paper we shall, unless otherwise stated, adopt the following terminology: $r=|z|, a=\left(1+r^{2}\right) /\left(1-r^{2}\right)$, $\rho=2 r /\left(1-r^{2}\right)$.

With a view to subsequent application we reproduce here the theorems of Zmorovič [6] and Sakaguchi [5].

THEOREM (ZMOROvič). Suppose $\psi(w, W)=M(w)+N(w) \cdot W$ where $M(w)$ and $N(w)$ are defined and finite in the half plane $\operatorname{Re} w>0$. Put

$$
w=\lambda_{1} \frac{1+z_{1}^{m}}{1-z_{1}^{m}}+\lambda_{2} \frac{1+z_{2}^{m}}{1-z_{2}^{m}}, \quad W=\lambda_{1} \frac{2 m z_{1}^{m}}{\left(1-z_{1}^{m}\right)^{2}}+\lambda_{2} \frac{2 m z_{2}^{m}}{\left(1-z_{2}^{m}\right)^{2}},
$$

Received by the editors June 9, 1971.

.AMS 1970 subject classifications. Primary 30A32.

Key words and phroses. Functions with positive real part, region of variability, variational formula.

(c) American Mathematical Society 1972 
where $z_{1}$ and $z_{2}$ are arbitrary points on $|z|=r<1, m$ is a positive integer, $\lambda_{1} \geqq 0, \lambda_{2} \geqq 0, \lambda_{1}+\lambda_{2} \equiv 1$. Then the function $\psi(w, W)$ can be represented as follows:

$$
\begin{aligned}
\psi(w, W)= & M(w)+(m / 2)\left(w^{2}-1\right) N(w) \\
& +(m / 2)\left(\rho^{2}-\rho_{0}^{2}\right) N(w) \cdots e^{2 i \psi}
\end{aligned}
$$

where $\quad\left(1+z_{k}^{m}\right) /\left(1-z_{k}^{m}\right)=a+\rho \exp \left(i \psi_{k}\right) \quad(k=1,2), \quad w=a+\rho_{0} \exp \left(i \psi_{0}\right)$ $\left(0 \leqq \rho_{0} \leqq \rho\right), \quad\left|z_{1}\right|=\left|z_{2}\right|=r, \quad a=\left(1+r^{2 m}\right) /\left(1-r^{2 m}\right), \quad \rho=2 r^{m} /\left(1-r^{2 m}\right), \quad e^{i \psi}=$ $i \exp \left(i\left(\psi_{1}+\psi_{2}\right) / 2\right)$.

COROLLARY. For fixed $w(|w-a|<\rho)$ the angle $2 \psi$ in the above formula can take all the values from $[0,2 \pi]$.

Theorem (SAKaguchi). Let $p(z) \in \mathscr{P}$. Then there exists a function $p^{*}(z)=p(z)+\delta p(z)$ belonging to $\mathscr{P}$ and with $\delta p(z)$ of the form

$$
\begin{aligned}
(2 / \lambda) \delta p(z)= & \varepsilon\left[p(z) \frac{1+\bar{\beta} z}{1-\bar{\beta} z}-(p(\beta))-\left(p(z)-\frac{1+\bar{\beta} z}{1-\bar{\beta} z}\right)-1\right] \\
& -\bar{\varepsilon}\left[p(z) \frac{\beta+z}{\beta-z}+p(\beta)\left(p(z)-\frac{\beta+z}{\beta-z}\right)-1\right]+o(1)
\end{aligned}
$$

where $\beta$ and $\varepsilon$ are arbitrary complex numbers such that $|\beta|<1,|\varepsilon|=1$ and $\lambda$ is a sufficiently small positive number.

\section{Extremal problems.}

THEOREM 1. Let $r, 0<r<1$, and $\alpha, 0 \leqq \alpha \leqq 2 \pi$, be given. If

$$
|\sin \alpha|<\left(2 a^{2}+1\right) \cdot\left(a^{2}+1\right)^{-3 / 2}
$$

then

$$
\min _{|z|=r} \min _{P \in \mathscr{P}} \operatorname{Re} e^{i \alpha}\left(z P^{\prime} \mid P\right)
$$

equals

$$
(y / 4 a)\left(\left(y^{2}-4\right)^{1 / 2} \cdot|\cos \alpha|+\left(4 a^{2}-y^{2}\right)^{1 / 2} \cdot|\sin \alpha|\right)
$$

where $y=-x^{1 / 2}$ and $x$ is the unique real root in $\left[4,4 a^{2}\right]$ of the equation

$$
\begin{aligned}
x^{3}-4\left(a^{2}+1\right) x^{2}+4\left(4 a^{2}+a^{4} \sin ^{2} \alpha+\cos ^{2} \alpha\right) x & \\
- & 16 a^{2}\left(\cos ^{2} \alpha+a^{2} \sin ^{2} \alpha\right)=0 .
\end{aligned}
$$

If

$$
|\sin \alpha| \geqq\left(2 a^{2}+1\right) \cdot\left(a^{2}+1\right)^{-3 / 2}
$$


then (3.2) equals

$$
|\sin \alpha|-\left(a^{2}+1\right)^{1 / 2}
$$

The above estimates are sharp.

Proof. Since $p\left(z e^{i \theta}\right) \in \mathscr{P}$ whenever $p(z) \in \mathscr{P}$ for any real $\theta$, we may assume that the minimum in (3.2) occurs at $z=r$. Since this minimum is, moreover, attained by a function of the form

$$
\lambda \frac{1+z \exp \left(-i \theta_{1}\right)}{1-z \exp \left(-i \theta_{1}\right)}+(1-\lambda) \frac{1+z \exp \left(-i \theta_{2}\right)}{1-z \exp \left(-i \theta_{2}\right)}
$$

where $\theta_{1}, \theta_{2}$ are arbitrary real constants in $[0,2 \pi]$ and $\lambda$ is a nonnegative number satisfying $0 \leqq \lambda \leqq 1$, we may apply (2.1) and obtain

$$
\operatorname{Re} e^{i \alpha} \frac{z P^{\prime}}{P}=\frac{1}{2} \operatorname{Re}\left[e^{i \alpha} \frac{P^{2}-1}{P}+e^{i \alpha} \frac{p^{2}-|P-a|^{2}}{P} e^{2 i \omega}\right] .
$$

Making use of the arbitrariness of $\psi$, we obtain

$$
\min \operatorname{Re} e^{i z} \frac{z P^{\prime}}{P}=\frac{1}{2} \operatorname{Re}\left[e^{i \alpha} \frac{P^{2}-1}{P}-\frac{\rho^{2}-|P-a|^{2}}{|P|}\right] \equiv \frac{1}{2} \psi_{P} .
$$

Equality holds in $\left(3.7^{\prime}\right)$ if

$$
\exp [i(2 \psi+\alpha-\phi)]=-1
$$

where $P=R e^{i \phi}$. Our problem now reduces to minimizing $\psi_{P}$ and we use Sakaguchi's variational formula to obtain the same. Let $p$ be the extremal function which at $z=r$ minimizes the right side of $\left(3.7^{\prime}\right)$. We have

$$
\begin{aligned}
& \psi_{p(r)}=\operatorname{Re}\left[\left(p-\frac{1}{p}\right) e^{i \alpha}-\frac{\rho^{2}-|p-a|^{2}}{|p|}\right] \\
& \psi_{p^{*}(r)}=\operatorname{Re}\left[\left(p+\delta p-\frac{1}{p+\delta p}\right) e^{i \alpha}-\frac{p^{2}-|p+\delta p-a|^{2}}{\mid p+\delta p !}\right]
\end{aligned}
$$

where $p^{*}=p+\delta p$ and $\delta p$ is given by (2.2). From (3.8) and (3.8') we obtain, after some simplification

$$
\begin{aligned}
& \psi_{p^{*}(r)}-\psi_{p(r)} \\
& =\operatorname{Re}\left[\left(1+\frac{1}{p^{2}}\right) e^{i \alpha}+\frac{1}{|p|}\left(2(\bar{p}-a)+\frac{\rho^{2}-|p-a|^{2}}{p}\right)\right] \delta p+o(1) .
\end{aligned}
$$

Let

$w=w(r)=\left(1+\frac{1}{p^{2}(r)}\right) e^{i \alpha}+\frac{1}{|p(r)|}\left(2((p(r))--a)+\frac{\rho^{2}-|p(r)-a|^{2}}{p(r)}\right)$. 
Substitution of the value of $\delta p$ from (2.2) in (3.9) yields

$$
\begin{aligned}
& \psi_{p^{*}(r)}-\psi_{p(r)} \\
& =\operatorname{Re}\left\{\frac { \lambda } { 2 } \overline { \varepsilon } \left[\left\{(p(r))-\frac{1+\beta r}{1-\beta r}-p(\beta)\left((p(r))-\frac{1+\beta r}{1-\beta r}\right)-1\right\} \bar{w}\right.\right. \\
& \left.\left.-\left\{p(r) \frac{\beta+r}{\beta-r}+p(\beta)\left(p(r)-\frac{\beta+r}{\beta-r}\right)-1\right\} w\right]+o(1)\right\}
\end{aligned}
$$

Since $p$ is extremal and $\varepsilon$ is arbitrary with $|\varepsilon|=1$, the expression within the square brackets in $\left(3.9^{\prime}\right)$ must vanish. This gives, on changing $\beta$ to $z$ that

$$
p(z)=A(z) / B(z),
$$

where

$$
\begin{aligned}
& A(z)=\left((p(r))-\frac{1+r z}{1-r z}-1\right) \bar{w}-\left(p(r) \frac{z+r}{z-r}-1\right) w, \\
& B(z)=\left((p(r))-\frac{1+r z}{1-r z}\right) \bar{w}+\left(p(r)-\frac{z+r}{z-r}\right) w,
\end{aligned}
$$

provided $B(z) \not \equiv 0$. But $B(z) \equiv 0$ only if $w=0$.

In case $w=0$ we obtain, on putting $p=R e^{i \phi}$ and equating the real and imaginary parts of $w$ to zero the equations

$$
\begin{aligned}
R^{2} \cos \alpha+\cos (\alpha-2 \phi)+2 R^{2} \cos \phi-2 a R & \\
& +\left(2 a R \cos \phi-1-R^{2}\right) \cos \phi=0, \\
R^{2} \sin \alpha+\sin (\alpha-2 \phi)-2 R^{2} & \sin \phi \\
- & \left(2 a R \cos \phi-1-R^{2}\right) \sin \phi=0
\end{aligned}
$$

from which we obtain

$$
\begin{aligned}
\tan \phi & =\frac{\left(1+R^{2}\right) \sin \alpha}{2 a R+\left(1-R^{2}\right) \cos \alpha}, \\
R^{2} & =\frac{1-\cos (\alpha-\phi)}{1+\cos (\alpha+\phi)}=\left(\frac{\sin ((\alpha-\phi) / 2)}{\cos ((\alpha+\phi) / 2)}\right)^{2} .
\end{aligned}
$$

Eliminating $R$ between (3.12), (3.12') we obtain

$$
\frac{\sin \phi}{\cos \phi \sin \alpha}=\frac{1-\sin \alpha \sin \phi}{a(\sin \alpha-\sin \phi)+\cos ^{2} \alpha \cos \phi}
$$

provided $\sin \alpha \geqq \sin \phi$. On simplification this yields

$$
(\sin \alpha-\sin \phi)(a \sin \phi-\cos \phi)=0
$$

so that either $\sin \phi=\sin \alpha$ or $\cot \phi=a$. Because of $\left(3.12^{\prime}\right)$ the former is 
ruled out and so we must have

$$
\cot \phi=a .
$$

Since always $|\tan \phi| \leqq \rho,(3.13)$ is possible only if $r+r^{-1} \leqq 5^{1 / 2}+i$, or equivalently, $\left(5^{1 / 2}+1-2^{1 / 2}\left(5^{1 / 2}+1\right)^{1 / 2}\right) / 2 \leqq r<1$. From the well-known result $\left|R e^{i \phi}-a\right| \leqq \rho$ we obtain

$$
\left(1+R^{2}\right) / 2 a R \leqq \cos \phi .
$$

Substituting the values of $R$ and $\phi$ from (3.12') and (3.13), (3.14) yields $\sin \alpha \geqq\left(2 a^{2}+1\right) \cdot\left(a^{2}+1\right)^{-3 / 2}$ where we have made use of the fact that $\sin \alpha>\sin \phi$. We note that $\left(2 a^{2}+1\right) \cdot\left(a^{2}+1\right)^{-3 / 2} \leqq 1$ if $r+r^{-1} \leqq 5^{1 / 2}+1$.

In case $\sin \alpha<\sin \phi$, we similarly obtain

$$
\cot \phi=-a,
$$

and $\sin \alpha \leqq-\left(2 a^{2}+1\right) \cdot\left(a^{2}+1\right)^{-3 / 2}$.

We have proved that if $w=0$ then $|\sin \alpha| \geqq\left(2 a^{2}+1\right) \cdot\left(a^{2}+1\right)^{-3 / 2}$. To prove the converse suppose, on the contrary, that $|\sin \alpha| \geqq\left(2 a^{2}+1\right)$ $\cdot\left(a^{2}+1\right)^{-8 / 2}$ and $w \neq 0$. Then $R$ and $\phi$ obtained from $\left(3.12^{\prime}\right)$ and (3.13), $\left(3.13^{\prime}\right)$ will not satisfy $(3.14)$ and so $|\sin \alpha|<\left(2 a^{2}+1\right) \cdot\left(a^{2}+1\right)^{-3 / 2}$.

To sum up:

If $|\sin \alpha| \geqq\left(2 a^{2}+1\right) \cdot\left(a^{2}+1\right)^{-3 / 2}$ then $w=0$.

If $|\sin \alpha|<\left(2 a^{2}+1\right) \cdot\left(a^{2}+1\right)^{-3 / 2}$ then $w \neq 0$.

We accordingly have the following two cases to consider:

Case I. $|\sin \alpha|<\left(2 a^{2}+1\right) \cdot\left(a^{2}+1\right)^{-3 / 2}$ so that $w \neq 0$. In this case $p(z)$ is given by (3.10). Since on $|z|=1, A(z)$ is pure imaginary and $B(z)$ is real, $p(z)$ is a rational function of $z$ satisfying $\operatorname{Re} p(z)=0$ on $|z|=1$. Hence $p(z)$ has no poles in $|z| \neq 1$ but has at least one pole on $|z|=1$. Moreover, all the poles of $p(z)$ must be simple [5]. It follows that $B(z)$ must have at least one zero on $|z|=1$, in view of the fact that $A(z)$ has no poles there.

Consider now the variation

$$
p^{*}(z)=\frac{p(z)+\lambda \phi(z)}{1+\lambda}=p(z)+\lambda(\phi(z)-p(z))+o(\lambda)
$$

where $\phi(z)=(1+\bar{\beta} z) /(1-\bar{\beta} z),|\beta|<1$ and $\lambda$ is a sufficiently small positive number. Then $p^{*}(z)$ belongs to $\mathscr{P}$ whenever $p(z) \in \mathscr{P}$.

For this variation we have

$$
\psi_{D^{*}(r)}-\psi_{p(r)}=\frac{\lambda}{2}\left[w\left(\frac{1+\bar{\beta} r}{1-\bar{\beta} r}-p(r)\right)+\bar{w}\left(\frac{1+\beta r}{1-\beta r}-(p(r))-\right)\right]+o(\lambda)
$$


Since $p$ is extremal, we must have

$$
\left((p(r))-\frac{1+\beta r}{1-\beta r}\right) \bar{w}+\left(p(r)-\frac{1+\bar{\beta} r}{1-\bar{\beta} r}\right) w \leqq 0 .
$$

Letting $|\beta| \rightarrow 1$, we must have $B(\beta) \leqq 0$ on $|\beta|=1$, that is,

$$
B(z) \leqq 0 \text { on }|z|=1 \text {. }
$$

From (3.15) we conclude that the zeros of $B(z)$ on $|z|=1$ are of even order. Since $B(z)=B_{1}(z) /(1-r z)(z-r)$ where $B_{1}(z)$ is a quadratic in $z$, it follows that $B(z)$ has a single zero of order two on $|z|=1$. Accordingly $p(z)$ must have the form

$$
p(z)=(1+\varepsilon z) /(1-\varepsilon z), \quad|\varepsilon|=1 .
$$

For such functions $p$ we have for $|z|=r$ the relation

$$
|p-a|=\rho \text {. }
$$

Putting $p=|p| e^{i \phi}$ and making use of (3.17) we obtain from (3.7'),

$$
\psi_{p}=\left(|p|-|p|^{-1}\right) \cos \phi \cos \alpha-\left(|p|+|p|^{-1}\right) \sin \phi \sin \alpha .
$$

Substituting the value of $\phi$ from (3.17) we get

$$
\begin{aligned}
& \min \psi_{p}=\min (2 a)^{-1}\left(|p|+|p|^{-1}\right) \\
& \cdot\left[\left(|p|-|p|^{-1}\right) \cos \alpha-|\sin \alpha|\left(4 a^{2}-\left(|p|+|p|^{-1}\right)^{2}\right)^{1 / 2}\right] .
\end{aligned}
$$

To obtain the minimum of this expression differentiate it with respect to $|p|$ and equate to zero. We obtain the equation

$$
\begin{aligned}
x^{3}-4\left(a^{2}+1\right) x^{2}+4\left(4 a^{2}+a^{4} \sin ^{2} \alpha\right. & \left.+\cos ^{2} \alpha\right) x \\
& -16 a^{2}\left(\cos ^{2} \alpha+a^{2} \sin ^{2} \alpha\right)=0
\end{aligned}
$$

where $x=y^{2}=\left(|p|+|p|^{-1}\right)^{2}$. After a long but straightforward calculation we find that this equation has only one real root provided $\sin ^{2} \alpha \leqq$ $\left(2 a^{2}-1\right)^{3} \cdot\left(a^{2}-1\right)^{-1} \cdot\left(a^{2}+1\right)^{-3}$. Since we are considering the case when $|\sin \alpha|<\left(2 a^{2}+1\right) \cdot\left(a^{2}+1\right)^{-3 / 2}$ the above condition is easily seen to be satisfied. That the real root lies in $\left[4,4 a^{2}\right]$ is readily verified. We, therefore, obtain the minimum value $\psi$ of $\frac{1}{2} \psi_{p}$,

$$
\psi=(y / 4 a)\left(\left(y^{2}-4\right)^{1 / 2} \cdot|\cos \alpha|+\left(4 a^{2}-y^{2}\right)^{1 / 2} \cdot|\sin \alpha|\right)
$$

where $y=-x^{1 / 2}$ and $x$ is the unique real root in $\left[4,4 a^{2}\right]$ of the cubic equation (3.4). This proves (3.3).

Case II. $|\sin \alpha| \geqq\left(2 a^{2}+1\right) \cdot\left(a^{2}+1\right)^{-3 / 2}$ so that $w=0$. Suppose first that $\sin \alpha>\sin \phi$. We have

$$
\min _{|z|=r} \min \operatorname{Re} e^{i \alpha} \frac{z P^{\prime}}{P}=\frac{1}{2}\left[\operatorname{Re}\left(p-\frac{1}{p}\right) e^{i x}-\frac{\rho^{2}-|p-a|^{2}}{|p|}\right]=\psi
$$


where $p=R e^{i \phi}$ and $R$ and $\phi$ are given by (3.12') and (3.13). Substituting the values of $R$ and $\phi$ we obtain $\psi=\sin \alpha-\left(a^{2}+1\right)^{1 / 2}$. In case $\sin \alpha<\sin \phi$ we have $\cot \phi=-a$ and obtain similarly $\psi=-\sin \alpha-\left(a^{2}+1\right)^{1 / 2}$.

In view of the fact that if $\sin \alpha>\sin \phi$ then $\sin \alpha>0$, and if $\sin \alpha<\sin \phi$ then $\sin \alpha<0$, the above two results may be combined to give (3.6).

We proceed to obtain the extremal function in case (3.5). Taking into consideration $\left(3.7^{\prime \prime}\right)$ and the fact that the minimum occurs for $p=R e^{i \phi}$ the extremal function is easily seen to be

$$
p(z)=\lambda \frac{1+z e^{i \theta}}{1-z e^{i \theta}}+(1-\lambda)\left(e^{i(\phi-\alpha)}\left(\frac{1+z e^{-i \theta}}{1-z e^{-i \theta}}-a\right)+a\right)
$$

where $\lambda$ and $\theta$ are obtained from the equations

$$
\lambda \frac{1+r e^{i \theta}}{1-r e^{i \theta}}+(1-\lambda)\left(e^{i(\phi-a)}\left(\frac{1+r e^{-i \theta}}{1-r e^{-i \theta}}-a\right)-a\right)=R e^{i \phi}
$$

where $R$ is given by equation (3.12') and $\phi$ is given by (3.13) if $\sin \alpha \geqq$ $\left(2 a^{2}+1\right) /\left(a^{2}+1\right)^{3 / 2}$ and by $\left(3.13^{\prime}\right)$ if $\sin \alpha \leqq-\left(2 a^{2}+1\right) /\left(a^{2}+1\right)^{3 / 2}$.

More generally extremal functions are of the type $p\left(z e^{i \gamma}\right)$ for arbitrary real $\gamma$.

THEOREM 2. Let $r, 0<r<1$, and $\alpha, 0 \leqq \alpha \leqq 2 \pi$, be given. If

$$
|\sin \alpha|<\left(2 a^{2}+1\right)\left(a^{2}+1\right)^{-3 / 2}
$$

then

$$
\max _{|z|=r} \max _{P_{\epsilon} \mathscr{Y}} \operatorname{Re} e^{i \alpha} \frac{z P^{\prime}}{P}
$$

equals

$$
(y / 4 a)\left(\left(y^{2}-4\right)^{1 / 2} \cdot|\cos \alpha|+\left(4 a^{2}-y^{2}\right)^{1 / 2} \cdot|\sin \alpha|\right)
$$

where $y=+x^{1 / 2}$ and $x$ is the unique real root in $\left[4,4 a^{2}\right]$ of the equation

$$
\begin{aligned}
x^{3}-4\left(a^{2}+1\right) x^{2}+4\left(4 a^{2}+a^{4} \sin ^{2} \alpha\right. & \left.+\cos ^{2} \alpha\right) x \\
& -16 a^{2}\left(\cos ^{2} \alpha+a^{2} \sin ^{2} \alpha\right)=0 .
\end{aligned}
$$

If

$$
|\sin \alpha .| \geqq\left(2 a^{2}+1\right)\left(a^{2}+1\right)^{-3 / 2}
$$

then (3.18) equals

$$
-|\sin \alpha|+\left(a^{2}+1\right)^{1 / 2} .
$$

The above estimates are sharp. 
Proof. Theorem 2 can be proved exactly as Theorem 1 . However, it follows directly from Theorem 1 by observing that if $p \in \mathscr{P}$ then $p^{-1}$ also belongs to $\mathscr{P}$. The estimates of Theorem 2 are sharp since the corresponding estimates of Theorem 1 are sharp.

Corollary. Let $P \in \mathscr{P}$. Then we have on $|z|=r, 0<r<1$,

$$
-\frac{2 r}{1-r^{2}} \leqq \operatorname{Re} \frac{z P^{\prime}}{P} \leqq \frac{2 r}{1-r^{2}}
$$

Proof. The results follow immediately from (3.3), (3.19) and (3.4) on putting $\alpha=0$.

ACKNowledgment. The author is thankful to Professor V. Singh for his help in the preparation of this paper.

\section{REFERENCES}

1. W. E. Kirwan II, Extremal problems for certain classes of analytic functions, Ph.D. Thesis, Rutgers University, New Brunswick, N. J., 1964.

2. R. J. Libera, Some radius of convexity problems, Duke Math. J. 31 (1964), 143-158. MR 28 \#4099.

3. M. S. Robertson, Variational methods for functions with positive real part, Trans. Amer. Math. Soc. 102 (1962), 82-93. MR 24 \#A3288.

4. - Extremal problems for analytic functions with positive real part and applications, Trans. Amer. Math. Soc. 106 (1963), 236-253. MR 26 \#325.

5. K. Sakaguchi, $A$ variational method for functions with positive real part, J. Math. Soc. Japan 16 (1964), 287-297. MR 31 \#1375.

6. V. A. Zmorovič, On bounds of convexity for starlike functions of order $\alpha$ in the circle $|z|<1$ and in the circular region $0<|z|<1$, Mat. Sb. 68 (110) (1965), 518-526; English transl., Amer. Math. Soc. Transl. (2) 80 (1969), 203-213. MR 33 \#5875.

Department of Mathematics, Punjabi University, Patiala, India 